\title{
Conhecimento produzido acerca da assistência de enfermagem às mulheres mastectomizadas: revisão integrativa
}

Recebido em: 14/03/2011

Aceito em: 27/07/2011

Ivete Maroso Krauzer ${ }^{1}$

Édlamar Kátia Adamy² Ana Rosa Cavalet ${ }^{3}$

Trata-se de uma revisão integrativa que sintetizou o conhecimento produzido acerca da assistência de enfermagem às mulheres mastectomizadas. Foram identificados o título, o método de pesquisa, o ano de publicação e os principais aspectos do conhecimento em oncologia. As fontes consultadas foram a Scientific Eletronic Library Online e a Biblioteca Virtual em Saúde. Foram selecionadas 22 produções escritas de 2005 a 2009. Os periódicos da Região Sudeste concentram mais produções, com 45,4\%. Utilizou-se o método qualitativo para abordar as dificuldades de enfrentamento ao câncer de mama e a mastectomia $(45,4 \%)$ e os diferentes aspectos da assistência de enfermagem.

Descritores: Cuidados de Enfermagem, Enfermagem Oncológica, Mastectomia.

Knowledge produced about nursing care to women mastectomies: integrative review

It is an integrative review that summarized the knowledge produced about the nursing care for women with mastectomies identified the title, method of research, publication year and the main aspects of knowledge in oncology. The sources consulted include the Scientific Electronic Library Online and Virtual Health Library. Selected were 22 written productions from 2005 to 2009. The journals of the Southeast more concentrated production, with $45.4 \%$. We used qualitative methods to address the difficulties of coping with breast cancer and mastectomy (45.4\%) and about the various aspects of nursing care.

Descriptors: Nursing care, Oncology Nursing, Mastectomy.

\section{Conocimiento producido sobre los cuidados a las mujeres mastectomizada: revisión integral}

Es un examen integrador que resume el conocimiento producido sobre los cuidados de enfermería para mujeres mastectomizadas. Se identificaron el título, el método de la investigación, el año de publicación y los principales aspectos de los conocimientos en oncología. Las fuentes consultadas incluyen la Scientific Electronic Library Online Biblioteca Virtual en Salud se seleccionó 22 producciones de 2005 a 2009. Los diarios de la producción del sudeste más concentrado, con $45,4 \%$. Se utilizaron los métodos cualitativos para hacer frente a las dificultades de lidiar con el cáncer de mama y la mastectomía $(45,4 \%)$ y sobre los diversos aspectos de los cuidados de enfermería.

Descriptores: Cuidados de Enfermería, Enfermería Oncológica, Mastectomía.

\section{INTRODUÇÃO}

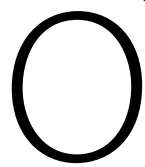
câncer tem representadoum problema desaúdepública, no Brasil e no mundo. As estatísticas mundiais mostram que, no ano 2000 , ocorreram 5,3 milhões de casos novos de câncer em homens e 4,7 milhões em mulheres, e que 6,2 milhões de pessoas morreram por essa causa (3,5 milhões de homens e 2,7 milhões de mulheres), correspondendo a $12 \%$ do total de mortes por todas as causas (cerca de 56 milhões) ${ }^{(1)}$.

A mama é o órgão apontado como segundo tipo de câncer mais frequente no mundo e o mais comum entre as mulheres. $A$ cada ano, cerca de $22 \%$ dos casos novos de câncer em mulheres são de mama ${ }^{(2)}$.

Esperava-se, para o Brasil, em 2010, um risco estimado de 49 casos a cada 100 mil mulheres. Isso representa uma incidência de 49.240 casos ao ano. Na Região Sudeste, o câncer de mama é o mais incidente entre as mulheres, com um risco estimado de
65 casos novos por 100 mil. Esse tipo de câncer também é o mais frequente nas mulheres das Regiões Sul (64/100.000), Centro-Oeste (38/100.000) e Nordeste (30/100.000). Na Região Norte, é o segundo tumor mais incidente $(17 / 100.000)^{(2)}$.

No estado de Santa Catarina, estima-se que, para cada 100 mil mulheres, existem 52,03 casos de câncer. Essas taxas colocam o estado em quinto lugar no país nos casos de neoplasias de mama, com taxas de incidências maiores do que a média nacional ${ }^{(3)}$.

Existem alternativas clínicas e cirúrgicas para tratamento. Os tratamentos clínicos convencionais são hormonioterapia, quimioterapia, radioterapia, os anticorpos monoclonais e as cirurgias conservadoras (tumorectomia, quadrantectomia)(4). As cirurgias não conservadoras são a adenomastectomia subcutânea ou mastectomia subcutânea. Na mastectomia simples ou total, ocorre a retirada da pele e do complexo aréolo papilar juntamente com a

1 Enfermeira Docente do Curso de Enfermagem da Universidade do Estado de Santa Catarina - UDESC e Universidade Comunitária da Região de Chapecó. - UNOCHAPECO.

2 Enfermeira Docente do Curso de Enfermagem da Universidade do Estado de Santa Catarina - UDESC.

3 Acadêmica da 8a Fase do Curso de Enfermagem da Universidade do Estado de Santa Catarina - UDESC. 
glândula mamária. A mastectomia radical modificada preserva um ou dois músculos peitorais com linfadenectomia axilar ${ }^{(4)}$.

As mastectomias apresentam-se como o procedimento invasivo mais utilizado para extirpar câncer de mama. Essa cirurgia pode causar traumas físicos e emocionais devido a sua complexidade, "exigindo cuidados de enfermagem específicos no pós-operatório. Além disso, no pós-cirúrgico, a enfermagem tem como papel ajudar a mulher na aceitação de sua nova imagem"(5:1556).

A imagem corporal é fortemente comprometida, despertando uma variação de sentimentos, especialmente o medo, na maioria das vezes relacionado à morte, e o preconceito da sociedade com sua imagem alterada. Muitas vezes, a própria mulher acaba rejeitando seu corpo, sentindo-se mutilada ${ }^{(6)}$.

Nesse contexto, a enfermagem desenvolve um papel importante, pois "o enfermeiro atua em ações de prevenção e controle. Tem como competência prestar assistência a pacientes com câncer na avaliação diagnóstica, tratamento, reabilitação e atendimento aos familiares"(7:615).

A atuação do enfermeiro se inicia logo após o diagnóstico, por meio da consulta de enfermagem a ser aplicada em cada etapa das atividades terapêuticas no período perioperatório. No momento da alta hospitalar, o enfermeiro encaminha a mulher para grupos de apoio interdisciplinar que discutem aspectos educativos, sociais e emocionais, visando à reintegração à vida cotidiana, e finaliza sua atuação no período de cicatrização(8:20)

O enfermeiro pode identificar a percepção da paciente sobre as alterações da imagem corporal e a alteração física da mama. A princípio, as pacientes podem se sentir desconfortáveis ao olhar a incisão cirúrgica. A despeito de quão preparada a paciente possa estar, a visão da incisão e da ausência da mama comumente é difícil para ela. "Explorar essa área sensível deve ser uma ação de enfermagem cuidadosa, e os indícios fornecidos pela paciente devem ser respeitados e manejados com sensibilidade."(5:1557)

\section{IDENTIFICAÇÃO DOTEMA}

De modo que essa variedade de tratamentos para o câncer de mama, as intervenções cirúrgicas, os índices epidemiológicos significativos no Brasil e no mundo e os sentimentos vivenciados pelas mulheres acometidas de câncer de mamas e submetidas à mastectomia despertou nestas pesquisadoras o interesse pela busca do conhecimento produzido sobre essa temática, estabelecemos como objetivo geral sintetizar o conhecimento produzido acerca da assistência de enfermagem às mulheres mastectomizadas.

\section{OBJETIVOS ESPECÍFICOS}

- Identificar os periódicos, teses ou dissertações com o título, método de pesquisa e ano de publicação;

- Conhecer quais os aspectos dessa área do conhecimento foram selecionadas pelos autores para realizar as pesquisas.

\section{METODOLOGIA}

Optamos pelo referencial de revisão integrativa que aponta seis etapas: a identificação do tema e seleção da hipótese ou questão de pesquisa (descrito na introdução); o estabelecimento de critérios para inclusão e exclusão de estudos; a definição das informações a serem extraídas dos estudos selecionados/categorização dos estudos; avaliação dos estudos incluídos na revisão integrativa; interpretação dos resultados e apresentação da revisão/síntese do conhecimento $^{(9)}$.

A primeira etapa (a identificação do tema) está descrita na introdução e as demais estão identificadas nos subtítulos a seguir, contemplando a metodologia proposta.

Estabelecimento de critérios para inclusão e exclusão de estudos O acesso e busca das informações foram realizados na Scientific Eletronic Library Online (SciELO) e Biblioteca Virtual em Saúde (BVS). Os descritores que estavam no projeto eram: "mulheres mastectomizadas", "cuidados de enfermagem" e "mastectomia". Durante a busca na literatura, foi necessário incluir o descritor "enfermagem oncológica".

A primeira busca nas fontes resultou na identificação de 94 produções que continham pelo menos um dos descritores apontados. Após uma leitura minuciosa dos resumos, foram selecionados 22 artigos. Excluímos os artigos que focavam em aspectos anátomo-clínicos das intercorrências cirúrgicas de mama, artigos escritos há mais de cinco anos e que não estavam em português.

\section{Definição das informações a serem extraídas dos estudos selecionados}

Em um primeiro momento, foram selecionados os resumos das produções. Nesses, foram identificados o nome de periódico ou tese, título do artigo, autores, ano de publicação, referencial metodológico e os aspectos da assistência de enfermagem que os autores selecionaram para desenvolver sua pesquisa. Foram considerados os trabalhos publicados de janeiro de 2005 a dezembro de 2009.

\section{RESULTADOS E DISCUSSÃO}

\section{Avaliação dos estudos incluídos na revisão integrativa}

Verificamos que as 94 produções foram encontradas em periódicos gerais da área de saúde. No Brasil, não existe"um periódico específico da enfermagem voltado para a cancerologia, e isso pode estar dificultando a divulgação do conhecimento produzido"(8:616).

As 22 produções selecionadas estão representadas no quadro 1 e significam a amostra que foi interpretada. A revisão integrativa, por ser a "mais ampla abordagem metodológica referente às revisões (...), combina também dados da literatura, além de incorporar um vasto leque de propósitos: definição de conceitos, revisão de teorias e evidências e análise de problemas metodológicos de um tópico particular"(10:103).

Constatamos que, no conjunto de obras, os periódicos da Região Sudeste (São Paulo, Barueri e Ribeirão Preto) foram os que mais publicaram artigos sobre a temática, com $45,4 \%$, seguidos dos da Região Centro-Oeste (Brasília e Goiás), com 31,8\%. A Região Sul, destacando Florianópolis, ficou com 22,8\% das produções publicadas. O maior número de artigos foi publicado no ano de 2008.

Os periódicos que mais publicaram foram a Revista Texto e Contexto Enfermagem (13,6\%) e a Nursing (13,6\%), seguidos da 


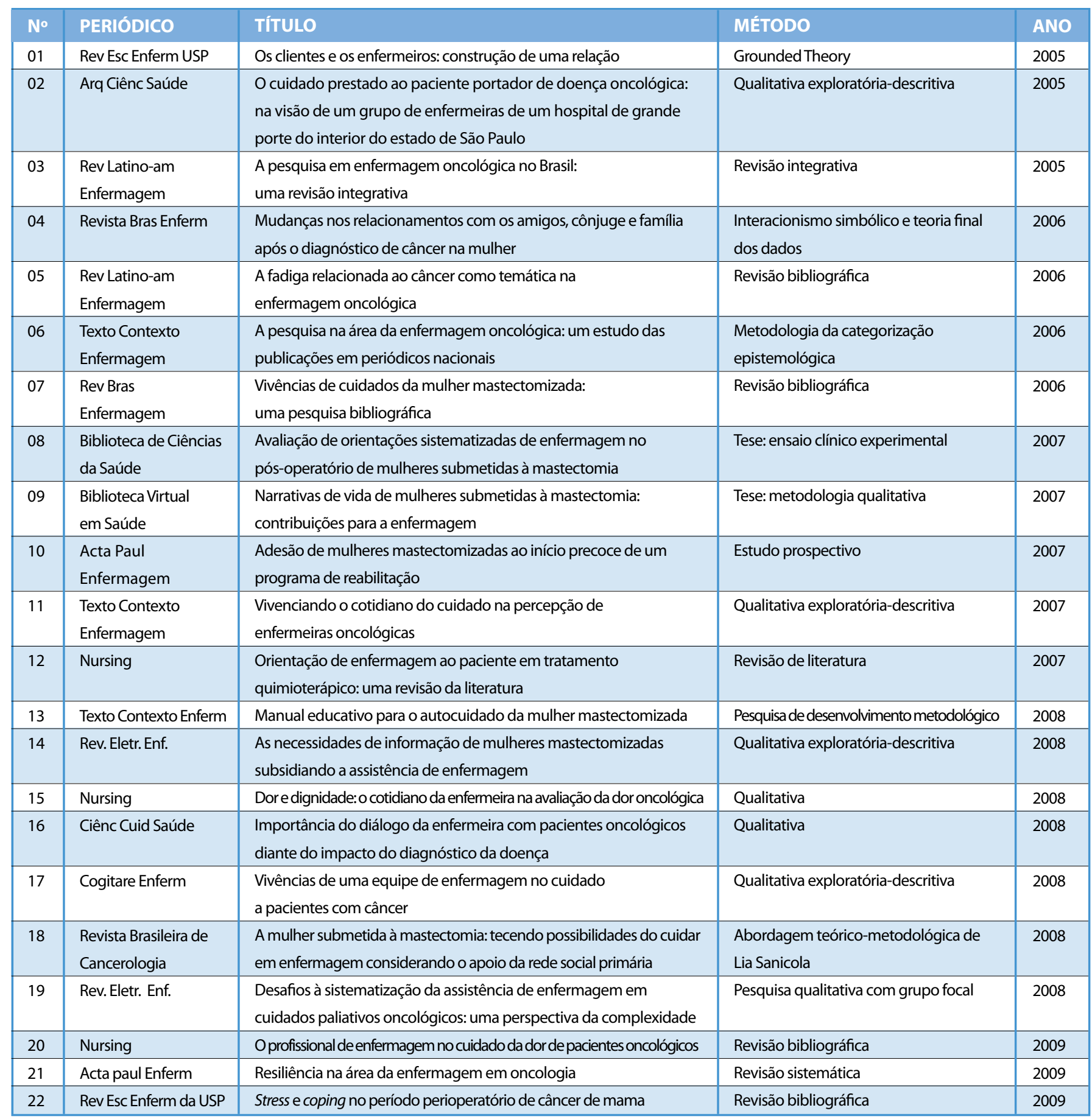

Quadro - Produções científicas selecionadas relativas aos anos de 2005 a 2009

Revista da Escola de Enfermagem da Universidade de São Paulo, Revista Brasileira de Enfermagem, Revista Eletrônica de Enfermagem, Acta Paulista e Revista Latino-Americana de Enfermagem, todas com 9\%. As revistas Ciência, Cuidado e Saúde, Revista Brasileira de Cancerologia, Cogitare Enfermagem, Arquivos de Ciências da Saúde publicaram um artigo individualmente, correspondendo a 4,5\%. Duas teses foram encontradas, sendo uma na Biblioteca de Ciências da Saúde e outra na Biblioteca Virtual de Saúde - BVS, com percentual de $4,5 \%$ para cada fonte.
Quanto aos métodos de pesquisa, foram identificados: cinco obras de revisão bibliográfica (22,7\%); quatro pesquisas qualitativas exploratório-descritivas $(18,1 \%)$; três pesquisas qualitativas, sem especificação do método (13,6\%); duas pesquisas qualitativas com grupo focal (9\%); uma revisão sistemática $(4,5 \%)$; uma revisão integrativa (4,5\%); um estudo prospectivo (4,5\%); o interacionismo simbólico foi tema de uma pesquisa $(4,5 \%)$; um ensaio clínico experimental $(4,5 \%)$; uma pesquisa de desenvolvimento metodológico (4,5\%); uma de categorização epistemológica 
(4,5\%); e uma obra que utilizou a Teoria Metodológica de Lia Sanícola (4,5\%).

Osaspectos delineadospelosautoresemsuasproduçõesversaram sobre vários aspectos da assistência. Encontramos dez artigos $(45,4 \%)$ que abordaram as dificuldades de enfrentamento à doença e à mastectomia, incluindo os sentimentos vivenciados (medo, angústia, ansiedade e culpa) pelas mulheres mastectomizadas; as diversas fases de enfrentamento do problema; os poucos estudos referentes ao aparecimento da fadiga; as mudanças que ocorrem nos relacionamentos familiares; a história de vida de mulheres mastectomizadas e a relação delas com as alterações que ocorreram em suas vidas, o stress e coping.

Onze artigos (50\%) abordaram as estratégias de intervenção utilizadas pela enfermagem, como educação em saúde, cuidados diante da dor oncológica, cuidados pós-operatórios, interação entre enfermeiros e pacientes submetidos à quimioterapia, conhecimento e análise dos meios de avaliação e registros realizados pela enfermagem, identificação da produção científica nacional e internacional sobre a resiliência na enfermagem em oncologia e discussão de sua aplicabilidade na assistência, criação de protocolo de assistência e aplicação da Sistematização da Assistência de Enfermagem (SAE) como estratégia de cuidados.

A construção de uma teoria de médio alcance (Grounded Theory), constituída de dois componentes - o de coleta de novos dados/ reavaliação do processo e acompanhamento da prestação de cuidados e o processo de intervenção terapêutica de enfermagem foi objeto de uma pesquisa, correspondendo a 4,5\%.

A classificação da produção do conhecimento na enfermagem oncológica entre 1994 e 2004 mostrou que são poucos os estudos que utilizam teorias de enfermagem como base teórica, e existe uma tendência para pesquisas qualitativas para a compreensão de vivências, emoções, sentimentos, qualidade de vida dos pacientes com câncer, permeando o processo de assistência oncológica(11:599).

Nesta revisão, notamos que prevaleceu o uso de metodologias qualitativas para o desenvolvimento das pesquisas. Nesse sentido, "a enfermagem oncológica brasileira, tal como a de outros países, tem pesquisado esse enfoque com o objetivo de desenvolver intervenções de enfermagem adequadas ao contexto sociocultural dos clientes"(7:617).

\section{CONCLUSÃO}

\section{Síntese do conhecimento}

Nesta revisão, foi possível identificar que o câncer de mama tem incidência e prevalência relevantes na sociedade brasileira e mundial. Essa realidade fez aumentar as cirurgias, como, por exemplo, a mastectomia.

Há diversidade de tratamentos e as mulheres enfrentam muitas dificuldades, gerando uma mudança em sua qualidade de vida. Um dos motivos para essa mudança é o sentimento que a mulher tem de estar mutilada. A mama, para as mulheres, está envolvida com a feminilidade, sexualidade e autoimagem. Alterações que ocorrem em qualquer desses fatores podem causar traumas físicos, emocionais e sociais.

A produção científica analisada mostra que a metade dos artigos escritos versou sobre os diferentes aspectos da assistência de enfermagem, seguida de produções sobre os sentimentos e dificuldades que as mulheres mastectomizadas enfrentam. Uma obra analisou os sentimentos dos profissionais de enfermagem frente ao atendimento de mulheres com câncer de mama.

A maioria dos artigos foi escrita por profissionais ligados às escolas da Região Sudeste, seguida da Região Centro-Oeste e Região Sul. Entretanto, se analisarmos por periódicos, encontramos a Revista Texto e Contexto (Região Sul) como uma das que mais publicaram artigos sobre esse tema.

Somente um artigo abordou a criação de protocolos de assistência. Foram encontrados estudos que trazem em comum à aplicação da sistematização de assistência de enfermagem. Desses estudos, a maioria aponta como resultado a importância de utilizar a sistematização como estratégia para o cuidado, mas infere o despreparo dos profissionais, por diversos motivos, para realizar a SAE.

A metodologia de revisão integrativa mostrou-se um instrumento importante para identificar o conhecimento produzido sobre a assistência de enfermagem às mulheres mastectomizadas.

\section{Referências}

1. Ministério da Saúde(BR), Instituto Nacional do Câncer - Inca. Câncer no Brasil: dados dos registros de base populacional. Brasília (DF): MS; 2010.

2. Ministério da Saúde (BR), Instituto Nacional do Câncer - Inca. Estimativa 2010

Incidência de câncer no Brasil. Brasilia (DF): MS; 2011.

3. Biazi CL, Proner C, Moreno M. Incidência de câncer de mama na região oeste

de Santa Catarina. In: Anais do I Congresso Interdisciplinar em saúde; 2010 abril

15-17: Chapecó, Brasil: Unochapecó - SC; 2010.

4. Tiezzi DG. Cirurgia conservadora no câncer de mama. Rev Bras de Ginecol

e Obstet. [online]. 2007 [acesso em 2010 out 10]; 29(8):428-434. Disponível

em: http://www.scielo.br/scielo.php?script=sci_arttext\&pid=\$0100-

72032007000800008>.

5. Smeltzer SC \& Bare BG. Tratado de enfermagem médico-cirúrgica. 10ª ed. Rio

de Janeiro (RJ): Guanabara-Koogan; 2005.

6. Pereira SG, Lunardi VC, Rosenhein DP, Bulhosa MS, Filho WDL. Vivências de

cuidados da mulher mastectomizada: uma pesquisa bibliográfica. Rev Bras de
Enferm. 2006; 59(6): 791-5.

7. Silveira CS, Zago MMF. Pesquisa brasileira em enfermagem oncológica: uma

revisăo integrativa. Rev Latino-am Enfermagem. 2006; 14 (4): 614.

8. Ministério da Saúde (BR). Instituto Nacional do Câncer - Inca. Controle do

câncer de mama: Documento de Consenso. Coordenação de Prevenção

e Vigilância. Brasilia (DF): MS; 2004

9. Mendes KDS, Silveira RCCP, Galvão CM. Revisão integrativa: método de

pesquisa para a incorporação de evidências na saúde e na enfermagem.

Texto Contexto Enferm. 2008; 17(4): 758-64.

10. Souza MT, Silva MD, Carvalho R. Revisão integrativa: o que é e como fazer. Einstein [on-line] 2010 [acesso 2011 fev 14]; 8(1):102-6. Disponível em: http:// apps.einstein.br/revista/arquivos/PDF/1134-Einsteinv8n1_p102-106_port.pdf. 11. Moreira MC, Camargo TC, Carvalho V, Figueiredo CF, Rosa LD, Bolzan MF. A pesquisa na área da enfermagem oncológica: um estudo das publicações em periódicos nacionais. Texto Contexto Enferm. 2006; 15(4): 595-600. 